\section{The Use of Hyaluronidase in Cosmetic Dermatology: A Review of the Literature}

\begin{abstract}
Background: Hyaluronidase can be employed to manage a variety of complications associated with cosmetic hyaluronic acid (HA) filler injection. However, the indications and treatment protocol for hyaluronidase use have not been well established.

Objective: Review of the available literature to describe the use of hyaluronidase in the reversal of $\mathrm{HA}$ filler injection.

Methods: PubMed/MEDUNE databases were utilized to identify case reports and studies pertaining to the use of hyaluronidase after HA filler injection.

Results: Hyaluronidase can be successfully employed in the management of uncomplicated nodules and overcorrection inflamed nodules, or tissue ischemia associated with HA filler injection. Hyaluronidase use is dependent on the clinical indication, anatomical location, and original injected HA quantity.

Conclusion: Hyaluronidase is an important tool for clinic ians utilizing cosmetic HA filler injection. Further reports and studies are warranted to firmly establish the ideal treatment protocol.
\end{abstract}

\section{Introduction}

Hyaluronic acid (HA) filler injection is an increasingly popular technique utilized for volume replacement, noninvasive skin rejuvenation, and soft tissue augmentation. Endogenous HA is a major structural component of the extracellular matrix of the skin and acts to maintain hydration in the dermis [1]. HA has an absorbent capacity one thousand times its volume. Filler injection with exogenous HA gel offers a number of advantages that contributes to its widespread use. HA is resorbable or biodegradable, typically over 6 to 18 months duration, and is associated with a less than one percent risk of hypersensitivity [1-4]. While HA injection is considered safe, several types of adverse events are recognized. If injections are placed too superficially or if excessive quantities are injected, there is a potential to develop the Tyndall effect, subcutaneous nodules, or asymmetrical outcomes. Other less common complications include: persistent edema, foreign body reactions, bacterial infection, tissue necrosis secondary to vascular occlusion or compression, and visual impairment from embolized filler material [1,4-6].

An additional benefit of HA is that it can be degraded through the use of hyaluronidase [3,7-9]. This is especially important in preventing some of the adverse outcomes aforementioned. Hyaluronidase is a naturally occurring enzyme and has been long used in medicine to facilitate the diffusion of anesthesia prior to ophthalmic procedures $[7,10]$. While the successful use of hyaluronidase to reverse the effects of exogenously injected HA has been described in a number of reports, there are currently no accepted standardized guidelines for its use. The dose, timing, injection technique, and reconstitution procedure are not currently well established or widely accepted [4].

\section{Journal of}

Clinical \& Investigative Dermatology

\author{
Brandon E. Cohen ${ }^{1}$, Sameer Bashey ${ }^{2}$ and Ashley \\ Wysong $^{2^{*}}$ \\ ${ }^{1}$ New York University School of Medicine, 550 First Avenue, New York, \\ NY 10001, USA \\ ${ }^{2}$ Department of Dermatology, Keck School of Medicine, University of \\ Southern California, 1441 Eastlake Avenue, Los Angeles, California, \\ 90033, USA

\section{*Address for Correspondence} \\ Ashley Wysong, Department of Dermatology, Keck School of Medicine, University \\ of Southern California, 1441 Eastlake Avenue, Los Angeles, California, 90033, \\ USA, Tel: (323)-442-0084; E-mail: Ashley.Wysong@med.usc.edu \\ Submission: 21 November 2015 \\ Accepted: 08 December 2015 \\ Published: 12 December 2015 \\ Copyright: (c) 2015 Cohen BE, et al. This is an open access article \\ distributed under the Creative Commons Attribution License, which permits \\ unrestricted use, distribution, and reproduction in any medium, provided the \\ original work is properly cited. \\ Reviewed \& Approved by: Dr. Kyoung-Chan Park, Professor, \\ Department of Dermatology, Seoul National University, Korea.
}

For example, the doses of hyaluronidase reported in the literature to reverse HA filler injection vary greatly from 5 to 300 units $[5,7,11]$.

Therefore we sought to review the available literature in order to better elucidate a standardized approach to the use of hyaluronidase after HA filler injection. PubMed/MEDLINE databases were utilized to identify studies pertaining to the use of hyaluronidase in the management of complications associated with HA filler injection. All available reports and studies from 1966 to present were considered in order to provide a comprehensive overview of the literature regarding the efficacy, safety, recommended use of hyaluronidase.

\section{Hyaluronidase Indications and Approach to Use}

The efficacy of hyaluronidase for the reversal of HA injections was formally demonstrated by a randomized, controlled trial conducted by Vartanian et al. [12]. In the study, twelve participants received two 0.2 $\mathrm{mL}$ injections of non-animal stabilized HA in the proximal forearm. One to three days after injection, skin scores were determined on a $0-5$ scale based on the size of augmentation. Participants then randomly received $0.5 \mathrm{~mL}$ of 75 units of hyaluronidase or normal saline vehicle. After one week, participants who received hyaluronidase demonstrated an $80 \%$ decline in skin scores, compared to a $10 \%$ decline among saline controls $(\mathrm{p}<0.001)$. Ninety days after treatment, there was no palpable remnant of the HA injection in $92 \%$ of subjects in the treatment group, while all control patients injected with saline continued to have detectable HA [12].

In practice, one primary consideration in the use of hyaluronidase is in the clinical context in which the removal of HA is desired. In general, complications of HA fillers can be categorized as emergent complications, notably vascular obstruction and skin necrosis, and non-emergent complications, such as over-correction, non-inflamed nodules, edema, and inflammatory nodules [4]. Accordingly the 
Citation: Cohen BE, Bashey S, Wysong A. The Use of Hyaluronidase in Cosmetic Dermatology: A Review of the Literature. J Clin Investigat Dermatol. 2015;3(2): 7 .

ISSN: 2373-1044

approach to using hyaluronidase should be adjusted according to the indication, anatomical location, and desired clinical effect $[7,13]$.

\section{Vascular obstruction and skin necrosis}

One uncommon but potentially serious complication of HA filler injection is skin necrosis [13]. It has been proposed that ischemia may occur secondary to compression of vasculature by extra-vascular filler material after the HA hydrates and expands, or through inadvertent intra-arterial HA injection $[3,6,8,14,15]$. There are also reports of skin necrosis in areas distant from the injection site, suggesting embolization after introduction of intra-arterial filler material $[3,16]$. One particularly ominous complication is the potential for visual impairment secondary to intra-arterial injection and obstruction of branches of the retinal or ophthalmic arteries $[4,17,18]$, which has most commonly been reported after filler injection into the glabella or nasolabial folds $[17,18]$.

Similarly, the most commonly reported injected areas associated with skin necrosis include the glabella and nasal ala $[3,4,19]$, as these regions have limited collateral blood supply $[6,8]$. Skin necrosis generally presents with blanching and dusky discoloration, along with pain in the affected area $[3,13]$. Venous occlusion has also been described, presenting with the delayed onset of vague discomfort and ecchymotic appearing lesions [4,20]. Management of ischemic complications may include the promotion of vasodilation through warm compress, $2 \%$ nitroglycerine paste, or sildenafil, as well as systemic corticosteroids, anticoagulation with aspirin or low molecular weight heparin, and intralesional hyaluronidase injection [3,8,19-21].

In this context, hyaluronidase, given in doses ranging from 30 75 units in normal saline or lidocaine, have been described $[8,14]$ While the exact timeframe for hyaluronidase injection has not been well established, hyaluronidase should be injected as early as possible. Hirsh and colleagues reported a case in which a patient developed signs of ischemia and impending tissue necrosis after injections into the nasolabial folds. The patient was successfully managed by the administration of 30 units of hyaluronidase in the region of suspected blockage six hours after the initial injection, along with two $325 \mathrm{mg}$ aspirin tablets, nitroglycerine paste, and warm compress [8]. In contrast, Kim et al. reported a case series of four patients who developed skin necrosis after HA filler injection in the nasal area. Two patients received hyaluronidase injection (dose undocumented) one day after the procedure, which failed in salvaging the affected skin [3]. In order to further elucidate the timing, Kim et al. conducted an experiment using rabbit ears in which HA filler was injected in the auricular arteries of five rabbits. Hyaluronidase was then injected at 4 -hour or 24-hour time points. The authors report that there was significant reduction in the area of necrosis when hyaluronidase was administered at the 4-hour time point, while no benefit was observed when injected at 24 hours [3].

When managing a case of intra-arterial HA injection studies suggest that direct intravascular administration of hyaluronidase is not typically required, as hyaluronidase readily diffuses into the vascular lumen $[3,13,22]$. DeLorenzi conducted a study in which fresh, intact human cadaveric facial arteries were filled with HA and placed in hyaluronidase solution. The authors reported elimination of luminal HA within four hours [22]. Therefore hyaluronidase may be injected into the region of a suspected obstruction rather than directly into the vasculature. Of note, DeLorenzi reports that through personal correspondence the author is aware of one unpublished case where ischemia resolved only after intra-arterial hyaluronidase injection [13]. While reports of intravascular hyaluronidase after filler injection were not identified, this technique has been historically described in the literature for other indications, such as for the treatment ulcers in the context of arterial disease [23].

\section{Non-inflamed lesions}

Excessive quantities or misplacement of HA may result in the development of subcutaneous nodules $[1,2,7,24]$. Given that HA is resorbable, uncomplicated nodules will eventually self-resolve over time [24]. However, if a nodule is painful or if the patient is bothered by its appearance, hyaluronidase can be employed to resolve the nodule. Several cases have been reported in the literature that describe successful resolution of nasojugal and cheek nodules associated with HA injection after injection of 75 units of hyaluronidase, reconstituted in $0.5 \%$ lidocaine with epinephrine $[25,26]$. However, several authors have advocated the use of lower initial doses. In one report, Hirsh and colleagues suggest that an initial injection of 30 units diluted in normal saline, along with follow up 3-4 days later is preferable [27].

In instances of overly superficial injection, nodules may form with a blue discoloration due to the "Tyndall effect", in which light scatters through the HA gel producing wavelengths that are perceived as blue $[8,17]$. Another potential adverse effect is the development of prolonged edema, especially in the malar region [21]. Management of persistent, defined as greater than one-month duration, malar edema consists of massage of the region, cold compress, systemic corticosteroid taper, as well as hyaluronidase injection [21]. Richards and colleagues reported a case describing the use of hyaluronidase for persistent, recurrent swelling two months after HA filler injection [9]. The patient was treated with 25 units of hyaluronidase prepared by dilution of 50 units $/ \mathrm{mL}$ hyaluronidase in bacteriostatic $0.9 \%$ saline. The patient presented two more times with the same complaint, at 4 and 18 months after initial injection, and was successfully treated with 25 units of hyaluronidase at each visit [9]. However, over that time period, one could conclude that the edema resolved as the HA product naturally resorbed. Hyaluronidase may also be used to manage complications associated with migration of filler material. In one case, a patient received $0.8 \mathrm{~mL}$ of $\mathrm{HA}$ in the cheeks bilaterally and developed intraorbital edema after three months. The authors hypothesized that the filler material may have migrated from the original injection location. Complete resolution was achieved after a single injection of 30 units of hyaluronidase into the lesion [9].

Given the inconsistency of doses in reported cases, Vartanian and colleagues sought to determine the necessary hyaluronidase dose in a prospective trial [12]. Eight participants received three injections with $0.2 \mathrm{~mL}$ of $\mathrm{HA}$ and after 3-5 days, each site was randomly injected with 10,20 or 30 units of hyaluronidase. A total volume of $0.4 \mathrm{~mL}$ was injected in all cases and hyaluronidase was reconstituted in normal saline. Upon follow up, there were no statistically significant differences between treated doses, although there was a nonsignificant trend towards more rapid decline of skin scores in lesions 
Citation: Cohen BE, Bashey S, Wysong A. The Use of Hyaluronidase in Cosmetic Dermatology: A Review of the Literature. J Clin Investigat Dermatol. 2015;3(2): 7

\section{ISSN: 2373-1044}

treated with higher doses. Based on these results, the authors suggest that 5-10 units of hyaluronidase administered in a volume of 0.1-0.2 $\mathrm{mL}(50$ units $/ \mathrm{mL})$ is an appropriate initial dose in non-emergent circumstances. It was additionally suggested that use of lower dose might mitigate the risk of local hypersensitivity [12]

\section{Periorbital lesions}

In areas of thinner skin, such as the inferior eyelids, lower doses of hyaluronidase have been successfully employed. Menon et al. reported the case of a patient who received $0.4 \mathrm{~mL}$ of $\mathrm{HA}$ in the lower lids and developed blue discoloration and evidence of overcorrection [11]. The

Table 1: Overview of reported cases and studies

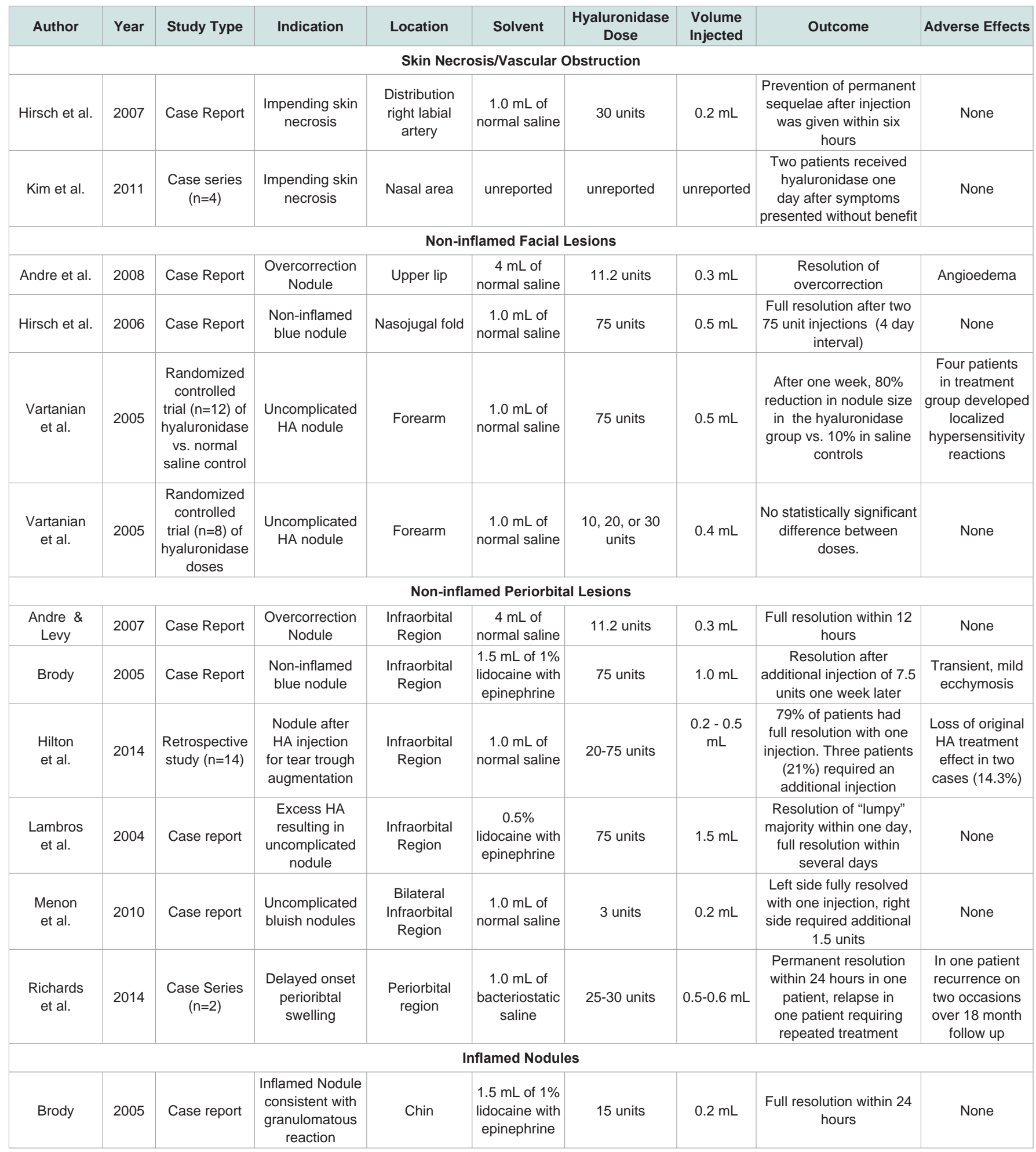


Citation: Cohen BE, Bashey S, Wysong A. The Use of Hyaluronidase in Cosmetic Dermatology: A Review of the Literature. J Clin Investigat Dermatol. 2015;3(2): 7 .

patient was initially treated with 3 units $(0.2 \mathrm{~mL}$ injected volume) of hyaluronidase reconstituted in normal saline into the affect regions. After one treatment, there was resolution of the left side, although the right side required an additional 1.5 units of hyaluronidase two days later [11]. While doses greater than 100 units have been reported for the management of uncomplicated overcorrection of the lower lids [28], the authors suggest that initial doses as low as 1.5-3 units are sufficient in this region and reduce the risk of allergic reactions and loss of initial treatment effect [11].

In a retrospective review of the management of lower eyelid edema following $\mathrm{HA}$ injection in 14 patients, hyaluronidase doses of 20-75 units (injected a volume of 0.2-0.5 mL) were injected per region [29]. All patients responded to therapy without known recurrence. In two cases, all previously injected HA was degraded, resulting in loss of treatment appearance. Accordingly, the authors advocate for starting at an initial lower dose than those reported in the study, followed by gradual increase in dose over multiple treatment sessions, if necessary [29].

The reconstitution of hyaluronidase in solution prior to injection has been suggested to facilitate diffusion and produce more rapid results than the injection of concentrated hyaluronidase [10]; however, there is heterogeneity among reported cases regarding the reconstitution solvent. Available reports describe the reconstitution of hyaluronidase in normal saline, lidocaine, or lidocaine with epinephrine, often without comment regarding the basis of the choice. In one case reported by Brody and colleagues, a patient presented with soft nodules with blue discoloration after HA injection in the bilateral infraorbital area [10]. The patient was treated with 75 units of hyaluronidase, given in a volume of $1 \mathrm{~mL}$ reconstituted in $1 \%$ lidocaine with epinephrine. Of note, the authors discuss lidocaine with epinephrine was selected with the aim of reducing bruising; however, this was ineffective, suggesting that dilution in normal saline or lidocaine is adequate [10].

\section{Inflammatory nodules}

The development of inflammatory nodules have also been described after HA injection and may occur due to infection and development of an active biofilm $[5,24]$. If infection is suspected, initial management may include oral antibiotics, incision and drainage if the lesion is fluctuant, and intralesional corticosteroids $[2,7,24]$. It is emphasized that steroids should also be administered after antibiotic treatment has been initiated [24]. Hyaluronidase injection has also been described in the management of painful, inflammatory nodules. Hyaluronidase has been demonstrated in vitro to effectively break down bacterial biofilms [30] and has been shown to have a role clinically in the management of infections related to filler injections [31]. Concurrent management with oral antibiotics is recommended as the administration of hyaluronidase may disseminate the injection by breaking up the collection [32].

Inflamed nodules may also occur due to granulomatous reactions associated with HA gel or contaminating proteins [25]. Brody et al. reported a case in which an inflamed nodule demonstrated to be sterile chronic granulomatous inflammation resolved using hyaluronidase. The patient received non-animal stabilized HA for perioral rhytides and one week later presented with an inflamed nodule [10]. Initial management with intralesional triamcinolone acetonide, an oral prednisone taper, and a course of cephalexin and trimethoprimsulfamethoxazole were ineffective. The nodule was then injected with 15 units of hyaluronidase in $1 \%$ lidocaine with epinephrine $(0.2 \mathrm{~mL}$ volume) and within one day the lesion permanently resolved (Table 1) [10].

\section{Adverse Effects of Hyaluronidase}

Although hyaluronidase is associated with a low risk of adverse effects, there have been reports of complications associated with its use, notably a risk of hypersensitivity reactions. In the trial by Vartanian and colleagues, four (25\%) patients developed localized hypersensitivity reactions characterized by transient erythema and pruritus, which developed on average thirty minutes after injection [12]. More severe hypersensitivity reactions, such as facial angioedema and anaphylaxis, have also been rarely described, with an estimated incidence of incidence of $0.1 \%$ [7,8,10,33]. However, document cases of anaphylaxis are associated with larger doses of utilized to facilitate anesthesia administration [34].

The risk of hypersensitivity is also related to the source of hyaluronidase employed. There are several commercially available types of hyaluronidase (Table2). These includehyaluronidase extracted from bovine testicular tissue (Amphadase ${ }^{\mathrm{TM}}$ and Hydase ${ }^{\mathrm{TM}}$ ), ovine testicular tissue (Vitrase ${ }^{\mathrm{TM}}$ ), or human recombinant hyaluronidase $\left(\right.$ Hylenex $\left.^{\mathrm{TM}}\right)$. The risk of allergic reaction is significantly reduced with the use of recombinant human hyaluronidase, compared to hyaluronidase from ovine or bovine sources $[7,35]$.

Andre et al. reported one case of angioedema occurring after a patient was treated with hyaluronidase for over correction of previous HA filler injection. The patient deferred allergy testing and

Table 2: Overview of commercially available hyaluronidase products

\begin{tabular}{|c|c|c|c|}
\hline Trade name & Source & Product Details & $\begin{array}{l}\text { Pregnancy } \\
\text { Category }\end{array}$ \\
\hline Amphadase $^{\mathrm{TM}}$ & Bovine Derived & $\begin{array}{c}150 \text { USP units per } \mathrm{mL} \text { in } 2 \mathrm{~mL} \\
\text { vial } \\
\text { Contains edetate disodium, } \\
\text { calcium chloride, monosodium } \\
\text { basic buffer, and thimerosal }\end{array}$ & C \\
\hline Hydase $^{T M}$ & Bovine Derived & $\begin{array}{c}150 \text { USP units per mL in } 2 \mathrm{~mL} \\
\text { vial } \\
\text { Contains sodium chloride, } \\
\text { edetate disodium, calcium } \\
\text { chloride, and monosodium } \\
\text { basic buffer }\end{array}$ & $\mathrm{C}$ \\
\hline Hylenex ${ }^{\mathrm{TM}}$ & $\begin{array}{l}\text { Human } \\
\text { Recombinant } \\
\text { Source }^{\#}\end{array}$ & $\begin{array}{l}150 \text { USP units per } \mathrm{mL} \text { in } 2 \mathrm{~mL} \\
\text { vial } \\
\text { Contains human albumin, } \\
\text { edetate disodium, and } \\
\text { polysorbate } 80\end{array}$ & $\mathrm{C}$ \\
\hline Vitrase $^{\mathrm{TM}}$ & Ovine Derived & $\begin{array}{l}200 \text { USP units per mL in } 2 \mathrm{~mL} \\
\text { vial } \\
\text { Contains lactose, potassium } \\
\text { phosphate dibasic buffer, } \\
\text { and potassium phosphate } \\
\text { monobasic buffer }\end{array}$ & $\mathrm{C}$ \\
\hline
\end{tabular}

*Wydase: Bovine derived. No longer commercially available \#Significantly reduced risk of hypersensitivity 
Citation: Cohen BE, Bashey S, Wysong A. The Use of Hyaluronidase in Cosmetic Dermatology: A Review of the Literature. J Clin Investigat Dermatol. 2015;3(2): 7

was then injected with 112.5 units of ovine-derived hyaluronidase and within ten minutes developed angioedema of the face [36]. The patient was successfully managed with betamethasone injection and a prednisolone taper [36]. While the large dose administered in this case may have contributed to the reaction, routine skin allergy testing prior to treatment with hyaluronidase has been advocated by several authors $[9,10,36,37]$. Brody et al. suggested intra-dermal injection of 3 units of hyaluronidase to test for the development of a wheal prior to hyaluronidase treatment, especially if derived from bovine or ovine sources [10]. However, in emergent cases of skin necrosis skin testing may not be practical [14].
Another consideration is that hyaluronidase is contraindicated in patients who have previously developed hypersensitivity reactions to bee or wasp stings $[8,13,25,36]$. Physicians should inquire about a history of allergy to insect stings, as cross reactivity has been demonstrated with endogenous hyaluronidase antigens [38]. It is also notable that certain medications, including aspirin, corticosteroids, estrogens, furosemide, benzodiazepines, phenytoinand antihistamines, may make tissues less sensitive to hyaluronidase and larger doses or repeated treatments may be necessary in patients taking these medications $[10,36]$.

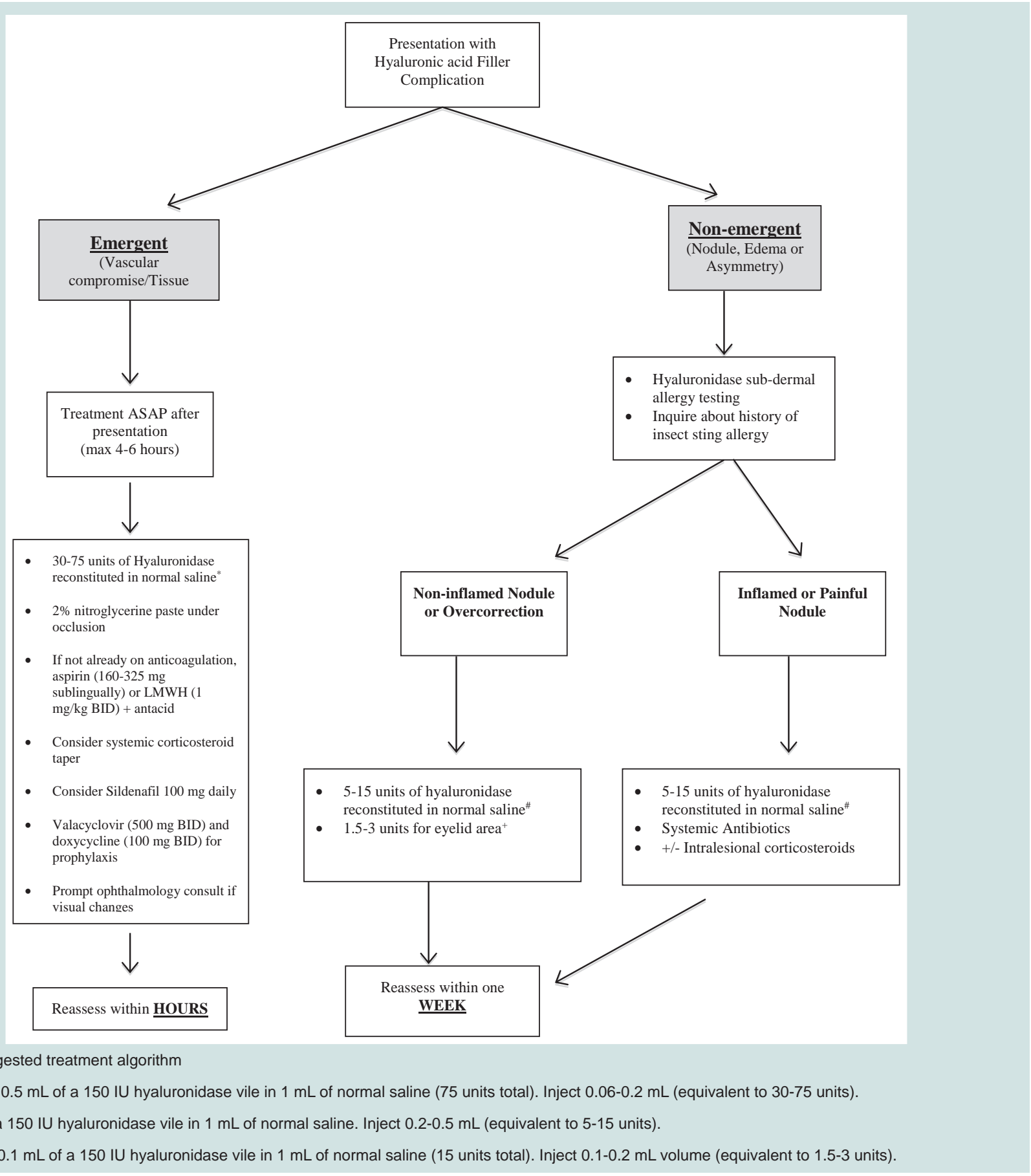


Citation: Cohen BE, Bashey S, Wysong A. The Use of Hyaluronidase in Cosmetic Dermatology: A Review of the Literature. J Clin Investigat Dermatol. 2015;3(2): 7

ISSN: 2373-1044

\section{Conclusions}

Hyaluronidase is an important tool in the management of complications related to HA filler injections. The recommended use and dosage of hyaluronidase depends on the clinical context and original quantity of HA administered. The majority of available types of hyaluronidase contain 150 units per $1 \mathrm{~mL}$, with the exception of Vitrase ${ }^{\mathrm{TM}}$, which is available as 200 units $/ \mathrm{mL}$. In the case of uncomplicated nodules involving periorbital, perioral, and nasal regions, 5-15 units may be initially attempted. For delicate areas such the lower eyelid, doses starting at 1.5-3 units may be employed. Similar starting doses may be utilized for inflammatory nodules, along with systemic antibiotics, followed by intralesional or systemic corticosteroids. In emergent cases of ischemia and impending skin necrosis, 30-75 units of hyaluronidase may be administered to the suspected region of blockage, along with warm compress, nitroglycerine, and, in patients who are not already taking anticoagulants, systemic anti-coagulation or anti-platelet agents. The direct intravascular injection of hyaluronidase is not generally required, as hyaluronidase has been shown to diffuse across vessel walls. After an ischemic event, systemic antibiotic and anti-herpetic prophylaxis is also recommended.

Evidence to support the use of lidocaine with or without epinephrine is lacking [32]. Therefore, normal saline can be considered to reconstitute the hyaluronidase (Figure 1). Given the potential for local and systemic allergic reactions associated with hyaluronidase, skin testing may be considered in non-emergent cases. The risk of hypersensitivity may be mitigated by the use of human recombinant hyaluronidase rather than animal derived sources.

As the popularity of HA filler injections continued to increase, hyaluronidaseuse can be expected to rise in parallel. Therefore continued report of cases and further prospective trials of hyaluronidase are warranted to continue to elucidate the indications and ideal treatment protocol.

\section{References}

1. Requena L, Requena $\mathrm{C}$, Christensen L, Zimmermann US, Kutzner $\mathrm{H}$, et al (2011) Adverse reactions to injectable soft tissue fillers. J Am Acad Dermatol 64: 1-34.

2. Bailey SH, Cohen JL, Kenkel JM (2011) Etiology, prevention, and treatment of dermal filler complications. Aesthet Surg J 31: 110-121.

3. Kim DW, Yoon ES, Ji YH, Park SH, Lee BI, et al. (2011) Vascular complications of hyaluronic acid fillers and the role of hyaluronidase in management. J Plast Reconstr Aesthet Surg 64: 1590-1595.

4. Park TH, Seo SW, Kim JK, Chang CH (2011) Clinical experience with hyaluronic acid-filler complications. J Plast Reconstr Aesthet Surg 64: 892896.

5. Ozturk CN, Li Y, Tung R, Parker L, Piliang MP, et al. (2013) Complications following injection of soft-tissue fillers. Aesthet Surg J 33: 862-877.

6. Kang MS, Park ES, Shin HS, Jung SG, Kim YB, et al. (2011) Skin necrosis of the nasal ala after injection of dermal fillers. Dermatol Surg 37: 375-380.

7. Cavallini M, Gazzola R, Metalla M, Vaienti L (2013) The role of hyaluronidase in the treatment of complications from hyaluronic acid dermal fillers. Aesthet Surg J 33: 1167-1174.

8. Hirsch RJ, Cohen JL, Carruthers JD (2007) Successful management of an unusual presentation of impending necrosis following a hyaluronic acid injection embolus and a proposed algorithm for management with hyaluronidase. Dermatol Surg 33: 357-360.
9. Richards AR (2014) Hyaluronidase. Aesthetics 1: 1-2.

10. Brody $\mathrm{HJ}$ (2005) Use of hyaluronidase in the treatment of granulomatous hyaluronic acid reactions or unwanted hyaluronic acid misplacement. Dermatol Surg 31(8 Pt 1): 893-897.

11. Menon H, Thomas M, D'Silva J (2010) Low dose of Hyaluronidase to treat over correction by HA filler--a case report. J Plast Reconstr Aesthet Surg 63: e416-e417.

12. Vartanian AJ, Frankel AS, Rubin MG (2005) Injected hyaluronidase reduces restylane-mediated cutaneous augmentation. Arch Facial Plast Surg 7: 231237.

13. DeLorenzi C (2014) Complications of injectable fillers, part 2: vascular complications. Aesthet Surg J 34: 584-600.

14. Grunebaum LD, Bogdan Allemann I, Dayan S, Mandy S, Baumann L (2009) The risk of alar necrosis associated with dermal filler injection. Dermatol Surg 35 Suppl 2: 1635-1640.

15. Inoue K, Sato K, Matsumoto D, Gonda K, Yoshimura K (2008) Arterial embolization and skin necrosis of the nasal ala following injection of dermal fillers. Plast Reconstr Surg 121: 127e-128e.

16. Schanz S, Schippert W, Ulmer A, Rassner G, Fierlbeck G (2002) Arterial embolization caused by injection of hyaluronic acid (Restylane). Br J Dermatol 146: 928-929.

17. Park SW, Woo SJ, Park KH, Huh JW, Jung C, et al. (2012) latrogenic retinal artery occlusion caused by cosmetic facial filler injections. Am J Ophthalmol 154: 653-662.e1.

18. Peter S, Mennel S (2006) Retinal branch artery occlusion following injection of hyaluronic acid (Restylane). Clin Experiment Ophthalmol 34: 363-364.

19. Glaich AS, Cohen JL, Goldberg LH (2006) Injection necrosis of the glabella: protocol for prevention and treatment after use of dermal fillers. Dermatol Surg 32: 276-281.

20. Sclafani AP, Fagien S (2009) Treatment of injectable soft tissue filler complications. Dermatol Surg 35 Suppl 2: 1672-1680.

21. Funt D, Pavicic T (2013) Dermal fillers in aesthetics: an overview of adverse events and treatment approaches. Clin Cosmet Investig Dermatol 6: 295-316.

22. DeLorenzi C (2014) Transarterial degradation of hyaluronic acid filler by hyaluronidase. Dermatol Surg 40: 832-841.

23. Castro CM, Grilli H, Grois J (1963) Intra-arterial hyaluronidase in the treatment of certain forms of lower limb ulcerations. Angiology 14: 277-284.

24. Narins RS, Coleman WP 3rd, Glogau RG (2009) Recommendations and treatment options for nodules and other filler complications. Dermatol Surg 35 Suppl 2: 1667-1671.

25. Hirsch RJ, Brody HJ, Carruthers JD (2007) Hyaluronidase in the office: a necessity for every dermasurgeon that injects hyaluronic acid. J Cosmet Laser Ther 9: 182-185.

26. Lambros $V$ (2004) The use of hyaluronidase to reverse the effects of hyaluronic acid filler. Plast Reconstr Surg 114: 277.

27. Hirsch RJ, Narurkar V, Carruthers J (2006) Management of injected hyaluronic acid induced Tyndall effects. Lasers Surg Med 38: 202-204

28. Pierre A, Levy PM (2007) Hyaluronidase offers an efficacious treatment for inaesthetic hyaluronic acid overcorrection. J Cosmet Dermatol 6: 159-162.

29. Hilton S, Schrumpf H, Buhren BA, Bolke E, Gerber PA (2014) Hyaluronidase injection for the treatment of eyelid edema: a retrospective analysis of 20 patients. Eur J Med Res 19: 30

30. Pecharki D, Petersen FC, Scheie AA (2008) Role of hyaluronidase in Streptococcus intermedius biofilm. Microbiology 154(Pt 3): 932-938.

31. Dayan SH, Arkins JP, Brindise R (2011) Soft tissue fillers and biofilms. Facial Plast Surg 27: 23-28.

32. Rzany B, Becker-Wegerich P, Bachmann F, Erdmann R, Wollina U (2009) Hyaluronidase in the correction of hyaluronic acid-based fillers: a review and 
Citation: Cohen BE, Bashey S, Wysong A. The Use of Hyaluronidase in Cosmetic Dermatology: A Review of the Literature. J Clin Investigat Dermatol. 2015;3(2): 7

ISSN: 2373-1044

a recommendation for use. J Cosmet Dermatol 8: 317-323.

33. Borchard K, Puy R, Nixon R (2010) Hyaluronidase allergy: a rare cause of periorbital inflammation. Australas J Dermatol 51: 49-51.

34. Ebo DG, Goossens S, Opsomer F, Bridts CH, Stevens WJ (2005) Flowassisted diagnosis of anaphylaxis to hyaluronidase. Allergy 60: 1333-1334.

35. Dunn AL, Heavner JE, Racz G, Day M (2010) Hyaluronidase: a review of approved formulations, indications and off-label use in chronic pain management. Expert Opin Biol Ther 10: 127-131.
36. Andre P. Flechet ML (2008) Angioedema after ovine hyaluronidase injection for treating hyaluronic acid overcorrection. J Cosmet Dermatol 7: 136-138.

37. Cohen JL (2008) Understanding, avoiding, and managing dermal filler complications. Dermatol Surg 34 Suppl 1: S92-S99.

38. Hemmer W, Focke M, Kolarich D, Dalik I, Gotz M, et al. (2004) Identification by immunoblot of venom glycoproteins displaying immunoglobulin E-binding $\mathrm{N}$-glycans as cross-reactive allergens in honeybee and yellow jacket venom. Clin Exp Allergy 34: 460-469. 